\title{
Magnetic Anomalies and the Corresponding Magnetic Centres. II
}

\author{
By Tsuneji RIKITAKE
}

Earthquake Research Institute

\begin{abstract}
The method described in the first report is extended to cases in which magnetic anomalies are given in dip.

The changes in magnetic dip that accompanied the Shizuoka Earthquake of 1935 are studied as an example.
\end{abstract}

1. Introduction. In the first report ${ }^{1)}$ of this paper, the writer proposed a method by which the position of magnetic centres of magnetic anomalies were directly determined from the observation of the vertical intensity of magnetic field on the earth's surface. The method will be extended here to cases in which magnetic anomalies are given in dip.

2. Magnetic anomalies in $X$ and $Y$. In a similar way with the treatment of anomalies in $Z$, the partial derivatives of $X$ and $Y$ (the northward and eastward components) at $x=y=0$ are calculated as follows;

$$
\begin{aligned}
& \frac{\partial^{j} X_{00}}{\partial x^{j-k} \partial y^{k}}=\frac{1}{2 \pi} \sum_{n m} \sum(-i)^{m+j+1} \frac{(n+j+1) !}{(n-m) !} a^{n+2} z^{-n-j-2}\left(a_{n}^{m} p_{j k k}^{m}+b_{n}^{m} q_{j k}^{m}\right), \\
& \frac{\partial^{j} Y_{00}}{\partial x^{j-k} \partial y^{k}}=\frac{1}{2 \pi} \sum_{n m} \sum_{m}(-i)^{m+j+1} \frac{(n+j+1) !}{(n-m) !} a^{n+2} z^{-n-j-2}\left(a_{n}^{m} r_{j k}^{m}+b_{n}^{m} s_{j k}^{m}\right),
\end{aligned}
$$

where $\left.\left.\quad \begin{array}{l}p_{j k}^{m} \\ q_{j k k}^{m}\end{array}\right\}=\int_{-\pi}^{\pi} \cos ^{j-k+1} u \sin ^{k} u \begin{array}{ll}\cos m u d u, & r_{j k}^{m} \\ s_{j k}^{m}\end{array}\right\}=\int_{-\pi}^{\pi} \cos ^{j-k} u \sin ^{k+1} u_{\sin }^{\cos } m u d u$.

$p, q, r$, and $s$ for $2 \leqq m$ and $j, k \leqq 3$ are calculated as given in Table I.

\begin{tabular}{|c|c|c|c|c|c|c|c|c|c|c|c|c|c|c|c|}
\hline$p^{m}$ & 0 & 1 & 2 & $q^{m}$ & 0 & 1 & 2 & $r^{m}$ & 0 & 1 & 2 & $s^{m}$ & 0 & 1 & 2 \\
\hline$p_{00}^{m}$ & 0 & $\pi$ & 0 & $q_{00}^{m}$ & 0 & 0 & 0 & $r_{00}^{m}$ & 0 & 0 & 0 & $s_{00}^{m}$ & 0 & $\pi$ & 0 \\
\hline$p_{10}^{m}$ & $\pi$ & 0 & $\frac{\pi}{2}$ & $q_{10}^{m}$ & 0 & 0 & 0 & $r_{10}^{m b}$ & 0 & 0 & 0 & $s_{10}^{m}$ & 0 & 0 & $\frac{\pi}{2}$ \\
\hline$p_{11}^{m}$ & 0 & 0 & 0 & $q_{11}^{m}$ & 0 & 0 & $\overline{2}$ & $r_{11}^{m}$ & $\pi$ & 0 & $-\frac{\pi}{2}$ & $s_{11}^{m}$ & 0 & 0 & 0 \\
\hline$p_{20}^{m}$ & 0 & $\frac{3}{4} \pi$ & 0 & $q_{20}^{m}$ & 0 & 0 & 0 & $r_{20}^{m}$ & 0 & 0 & 0 & $s_{20}^{m}$ & 0 & $\frac{\pi}{4}$ & 0 \\
\hline$p_{21}^{m}$ & 0 & 0 & 0 & $q_{21}^{m}$ & 0 & $\frac{\pi}{4}$ & 0 & $r_{21}^{m e}$ & 0 & $\frac{\pi}{4}$ & 0 & $s_{21}^{m}$ & 0 & 0 & 0 \\
\hline$p_{22}^{m}$ & 0 & $\frac{\pi}{4}$ & 0 & $q_{22}^{m}$ & 0 & 0 & 0 & $r_{22}^{m}$ & 0 & 0 & 0 & $s_{22}^{m}$ & 0 & $\frac{3}{4} \pi$ & 0 \\
\hline$p_{30}^{m}$ & $\frac{3}{4} \pi$ & 0 & $\frac{\pi}{2}$ & $q_{30}^{m}$ & 0 & 0 & 0 & $r_{30}^{m}$ & 0 & 0 & 0 & $s_{30}^{m}$ & 0 & 0 & $\frac{\pi}{4}$ \\
\hline$p_{31}^{m}$ & 0 & 0 & 0 & $q_{31}^{m}$ & 0 & 0 & $\frac{\pi}{4}$ & $r_{31}^{m}$ & $\frac{\pi}{4}$ & 0 & 0 & $s_{31}^{m}$ & 0 & 0 & 0 \\
\hline$p_{32}^{m}$ & $\frac{\pi}{4}$ & 0 & 0 & $q_{32}^{m}$ & 0 & 0 & 0 & $r_{32}^{m}$ & 0 & 0 & 0 & $s_{32}^{m}$ & 0 & 0 & $\frac{\pi}{4}$ \\
\hline$p_{33}^{m}$ & 0 & 0 & 0 & $q_{33}^{m}$ & 0 & 0 & $\frac{\pi}{4}$ & $r_{33}^{m}$ & $\frac{3}{4} \pi$ & 0 & $-\frac{\pi}{2}$ & $s_{33}^{m}$ & 0 & 0 & 0 \\
\hline
\end{tabular}

Table I 
Accordingly, we have the next relations for the cases $2 \geq n$

$$
\begin{aligned}
& \begin{array}{lll}
-a_{1}{ }^{1}-\sqrt{3} a_{2}{ }^{1} & =X_{00} \\
-3 a_{1}{ }^{0}-6 a_{2}{ }^{0}+\sqrt{3} a_{2}{ }^{2} & =a \partial X_{00} / \partial x & , \\
\sqrt{3} b_{2}{ }^{2} & =a \partial X_{00} / \partial y & , \\
9 a_{1}{ }^{1}+15 \sqrt{3} a_{2}{ }^{1} & =a^{2} \partial^{2} X_{00} / \partial x^{2} & , \\
3 b_{1}{ }^{1}+5 \sqrt{3} b_{2}{ }^{1} & =a^{2} \partial^{2} X_{00} / \partial x \partial y \quad, \\
3 a_{1}{ }^{1}+5 \sqrt{3} a_{2}{ }^{1} & =a^{2} \partial^{2} X_{00} / \partial y^{2}, \\
45_{1}{ }^{0} a+135 a_{2}{ }^{0}-30 \sqrt{3} a_{2}{ }^{2} & =a^{3} \partial^{3} X_{00} / \partial x^{3}, \\
-15 \sqrt{3} b_{2}{ }^{2} & & a^{3} \partial^{3} X_{00} / \partial x^{2} \partial y, \\
15 a_{1}{ }^{0}+45 a_{2}{ }^{0} & & =a^{3} \partial^{3} X_{00} / \partial x \partial y^{2}, \\
-15 \sqrt{3} b_{2}{ }^{2} & & =a^{3} \partial^{3} X_{00} / \partial y^{3},
\end{array}, \\
& \left.\begin{array}{lll}
-b_{1}{ }^{1}-\sqrt{3} b_{2}{ }^{1} & =Y_{00} & ; \\
\sqrt{3} b_{2}{ }^{2} & =a \partial Y_{00} / \partial x & , \\
-3 a_{1}{ }^{0}-6 a_{2}{ }^{0}-\sqrt{3} a_{2}{ }^{2} & =a \partial Y_{00} / \partial y & , \\
3 b_{1}{ }^{1}+5 \sqrt{3} b_{2}{ }^{1} & =a^{2} \partial^{2} Y_{00} / \partial x^{2} & , \\
3 a_{1}{ }^{1}+5 \sqrt{3} a_{2}{ }^{1} & =a^{2} \partial^{2} Y_{00} / \partial x \partial y, \\
9 b_{1}{ }^{1}+15 \sqrt{3} b_{2}{ }^{1} & =a^{2} \partial^{2} Y_{00} / \partial y^{2} \quad, \\
-15 \sqrt{3} b_{2}{ }^{2} & =a^{3} \partial^{3} Y_{00} / \partial x^{3}, \\
15 a_{1}{ }^{0}+45 a_{2}{ }^{0} & =a^{3} \partial^{3} Y_{00} / \partial x^{2} \partial y, \\
-15 \sqrt{3} b_{2}{ }^{2} & =a^{3} \partial^{3} Y_{00} / \partial x \partial y^{2}, \\
45 a_{1}{ }^{0}+135 a_{2}{ }^{0}+30 \sqrt{3} a_{2}{ }^{2} & =a^{3} \partial^{3} Y_{00} / \partial y^{3},
\end{array}\right)
\end{aligned}
$$

and

where it is assumed that $a=z$ as before.

By solving these simultaneous equations (3) and (4) respectively, we can determine $a_{1}{ }^{0}, a_{1}{ }^{1}, \ldots$, where it must be assumed that $X_{00}, \partial X_{00} / \partial x, \ldots, X_{00}, \partial Y_{00} / \partial x, \ldots$ are already given from Taylor expansions of $X$ and $Y$. In order to determine the magnetic centre, the parameter $a$ should be adjusted then so as to make the potential due to the second degree terms minimum as studied in the case of $Z$.

3. Magnetic anomalies in dip. Among the absolute measurements of the elements of magnetic field, the measurement of dip is comparatively easy. Hence it is importance for practical use to analyze magnetic anomalies in dip.

If it is supposed that the anomalies in dip are not large, we may take the relation

$$
\tan \left(\theta_{n}+\Delta \theta\right) \div \tan \theta_{n}+\tan \theta_{n}\left(\frac{\Delta Z}{Z_{n}}-\frac{\Delta X}{X_{n}}\right),
$$

where $X_{n}$ and $Z_{n}$ denote respectively the northward and downward components of the normal magnetic field, $\theta_{n}$ the normal values of dip, $\Delta X, \Delta Z$ and $\Delta \theta$ denote their changes.

Then, by putting

$$
f(\Delta \theta)=\frac{\tan \left(\theta_{n}+\Delta \theta\right)-\tan \theta_{n}}{\tan \theta_{n}}
$$

we have the relation 


$$
f(\Delta \theta)=\frac{\Delta Z}{Z_{n}}-\frac{\Delta X}{X_{n}} .
$$

Combining (6) of the first paper with (3) of the present paper, we can easily get the next relation

$$
\begin{aligned}
& \frac{1}{Z_{n}}\left(2 a_{1}{ }^{0}+3 a_{2}{ }^{0}\right)+\frac{1}{X_{n}}\left(a_{1}{ }^{1}+\sqrt{3} a_{2}{ }^{1}\right) \\
& -\frac{1}{Z_{n}}\left(3 a_{1}{ }^{1}+4 \sqrt{3} a_{2}{ }^{1}\right)+\frac{1}{X_{n}}\left(3 a_{1}{ }^{0}+6 a^{z 0}-\sqrt{3} a_{2}{ }^{2}\right) \\
& -\frac{1}{Z_{n}}\left(3 b_{1}^{1}+4 \sqrt{3} b_{2}^{1}\right)-\frac{1}{X_{n}} \sqrt{3} b_{2}^{2} \\
& -\frac{{ }^{\prime} \frac{1}{Z_{n}}}{}\left(12 a_{1}{ }^{0}+30 a_{2}{ }^{0}-5 \sqrt{3} a_{2}{ }^{2}\right)-\frac{1}{X_{a}}\left(9 a_{1}{ }^{1}+15 V \overline{3} a_{2}{ }^{1}\right)=a^{2} \partial^{2} f_{00} / \partial x^{2} \\
& -\frac{1}{Z_{n}} 5 \sqrt{3} b_{2}{ }^{2}-\frac{1}{X_{n}}\left(3 b_{1}{ }^{1}+5 \sqrt{3} b_{2}{ }^{1}\right) \quad=a^{2} \partial^{2} f_{00} / \partial x \partial y, \\
& -\frac{1}{Z_{n}}\left(12 a_{1}^{0}+30 a_{2}{ }^{0}+5 \sqrt{3} a_{2}{ }^{2}\right)-\frac{1}{X_{n}}\left(3 a_{1}{ }^{1}+5 \sqrt{3} a_{2}{ }^{1}\right)=a^{2} \partial^{2} f_{00} / \partial y^{2}, \\
& -\frac{1}{Z_{n}}\left(45 a_{1}{ }^{1}+90 \sqrt{3} a_{2}{ }^{1}\right)-\frac{1}{X_{n}}\left(45 a_{1}{ }^{0}+135 a_{2}{ }^{0}-30 \sqrt{3} a_{2}{ }^{2}\right)=a^{3} \partial^{3} f_{00} / \partial x^{3}, \\
& -\frac{1}{Z_{n}}\left(15 b_{1}{ }^{1}+30 \sqrt{3} b_{2}{ }^{1}\right)+\frac{1}{X_{n}} 15 \sqrt{3} b_{2}{ }^{2} \quad=a^{3} \partial^{3} f_{00} / \partial x^{2} \partial y, \\
& -\frac{1}{Z_{n}}\left(15 a_{1}{ }^{1}+30 \sqrt{3} a_{2}{ }^{1}\right)-\frac{1}{X_{n}}\left(15 a_{1}{ }^{0}+45 a_{2}{ }^{0}\right) \quad=a^{3} \partial^{3} f_{00} / \partial x \partial y^{2} \text {, } \\
& -\frac{1}{Z_{n}}\left(45 b_{1}{ }^{1}+90 \sqrt{3} b_{2}{ }^{2}\right)+\frac{1}{X_{n}} 15 \sqrt{3} b_{2}{ }^{2} \quad=a^{3} \partial^{3} f_{00} / \partial y^{3}
\end{aligned}
$$

The coefficients of the spherical hprmonic expansion are determined as the solutions of these simultaneous equations.

4. Actual example. Y. Kato ${ }^{2)}$ observed repeatedly the changes in magnetic dip in the vicinity of the epicentral area after the Shizuoka Earthquake of July 11, 1935. The differences of the values obtained in the survey in Aug. from that in July are

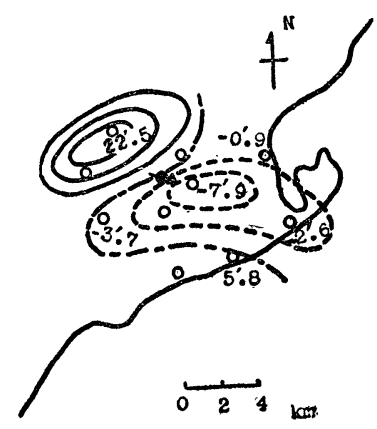

The changes in dip after the Shizuoka Earthquake. (After Y. Kato) reproduced in Fig. 1. In like manner with the treatment in the case of Volcano Miyakesima in the first report, $\Delta \theta$ was read off from the map from which $f_{00}, \partial f_{00} / \partial x, \ldots$ are calculated by means of least square. From the relation $Z_{0}=0$, the parameter $a$ was determined to be $4.2 \mathrm{~km}$. The position of the magnetic centre or dipole was marked with a small circle on the map. The north seeking end of the dipole directs toward $\mathrm{E} 7^{\circ} \mathrm{S}$. Its dip is also obtained to be $7^{\circ}$. The moment amounts to $4.4 \times 10^{15} \mathrm{emu}$. The result agreed well with Kato's study in which he determined the position, direction and intensity of the corresponding dipole by means of trial and error method. However, it is not clear why the changes in geomagnetism as obtained here took place in connexion with earthquake occurrence, 


\section{Conclusion}

The writer showed in this paper that the position, direction and intensity of a subterranean magnetic dipole can be found directly from the anomalies in dip as well as in $Z$ that was already studied in the first report. Applying the method to the anomalies in dip in the case of Shizuoka Earthquake of July, 1935, some interesting results were obtained.

(Read : May 22, 1950)

\section{References}

1) T. Rikitake, Journ. Geomag. Geoelectr. 2, 20 (1950).

2) Y Kato. Sci. Rep. Tohoku Univ. 27, 1 (1939). 